\title{
Improvement of 1,3-Propanediol Production From Crude Glycerol by Co-cultivation of Anaerobic and Facultative Microbes Under Non-strictly Anaerobic Conditions
}

\author{
Yaqin Sun ( $\nabla$ sunyaqin@dlut.edu.cn ) \\ Dalian University of Technology \\ Lingyun Liang \\ Dalian University of Technology \\ Yafeng Zheng \\ Dalian University of Technology \\ Jindong Han \\ Dalian University of Technology \\ Zhilong Xiu \\ Dalian University of Technology
}

\section{Research}

Keywords: Synthetic microbial consortium, 1,3-propandiol, crude glycerol, Clostridium butyricum, Klebsiella pneumoniae

Posted Date: November 23rd, 2021

DOI: https://doi.org/10.21203/rs.3.rs-1095907/v1

License: (c) (i) This work is licensed under a Creative Commons Attribution 4.0 International License.

Read Full License 


\section{Abstract}

Background: Natural microbial consortia could efficiently produce 1,3-propanediol, the most promising bulk biochemical derived from glycerol that can be used as a monomer in the synthesis of polytrimethylene terephthalate (PTT). While natural microbial communities are made up of a diverse range of microbes with frequently unknown functions, the construction of synthetic microbial consortia allows for creating more defined systems with lower complexity.

Results: In this study, the synthetic microbial consortia were constructed by combing facultative microbes of Klebsiella pneumoniae DUT2 (KP) and/or Escherichia coli DUT3 (EC) cultures with the strict anaerobic microbe of Clostridium butyricum DUT1 (CB) cultures under micro-aerobic conditions. The function of EC and KP during the fermentation process was to deplete oxygen and provide an anaerobic environment for $\mathrm{CB}$. Furthermore, KP competes with CB to consume crude glycerol and produce 1,3-PDO. The interaction of commensalism and competition resulted in synthetic microbial consortia that could efficiently convert crude glycerol to 1,3-PDO even under micro-aerobic conditions. In a batch fermentation, the synthetic $\mathrm{CB}: \mathrm{KP}$ co-culture at an initial abundance ratio of 92.5:7.5 yielded a maximum 1,3-PDO concentration of $52.08 \mathrm{~g} / \mathrm{L}$, with a yield of $0.49 \mathrm{~g} / \mathrm{g}$ and a productivity of $1.80 \mathrm{~g} /(\mathrm{L} . \mathrm{h})$, which increased by $10 \%, 9 \%$, and $12 \%$, respectively, when compared to the $\mathrm{CB}$ mono-culture under strictly anaerobic conditions. Compared to the KP mono-culture, the final 1,3-PDO concentration, yield, and productivity by the synthetic $\mathrm{CB}: \mathrm{KP}$ consortia increased by $16 \%, 19 \%$, and $84 \%$, respectively. The synthetic CB:KP:EC co-culture achieved the highest 1,3-PDO flux of $49.17 \%$ at an initial abundance ratio of $85: 7.5: 7.5$, while $7.43 \%, 5.77 \%, 3.15 \%$ $4.24 \%$, and $2.13 \%$ of flux was distributed to butyric acid, acetic acid, lactic acid, ethanol, and succinic acid pathways. In a fed-batch fermentation, synthetic CB:KP:EC co-culture demonstrated a maximum 1,3-PDO concentration of $77.68 \mathrm{~g} / \mathrm{L}$ with a yield of $0.51 \mathrm{~g} / \mathrm{g}$ which is $30 \%$ and $13 \%$ higher than the production by the $\mathrm{CB}$ mono-culture at $0.02 \mathrm{vvm} \mathrm{N}_{2}$ supply. The initial abundance of $\mathrm{CB}$ guaranteed to be at least $85 \%$ facilitates 1,3-PDO production from crude glycerol efficiently by the development of synthetic microbial consortia.

Conclusion: Under micro-aerobic conditions, the synthetic microbial consortia demonstrated excellent performance on 1,3-propanediol production via the interaction of commensalism and competition. The experimental results demonstrated the potential benefit of using the synthetic microbial consortia to produce 1,3-propanediol from crude glycerol.

\section{Background}

Since thousands of years, microbial consortia have been widely used in traditional food and beverage fermentation due to their robustness against environmental fluctuations and excellent performances for complex tasks [1,2]. The use of natural microbial communities in the bioconversion of raw materials to biochemicals such as 1,3-propanediol, lactic acid, ethanol, and propionate has recently received a lot of attention [3-6]. All of these microbial consortia demonstrate excellent utilization of complex carbon sources and high efficiency in producing target products. 
In order to improve raw glycerol metabolism to 1,3-propanediol (1,3-PD0), the most promising bulk biochemical that can be used as a monomer for the synthesis of polytrimethylene terephthalate (PTT), several studies focusing on natural microbial consortium has been developed [4, 7-12]. In fed-batch cultivation, a mixed culture from a municipal wastewater treatment plant produced $70.0 \mathrm{~g} / \mathrm{L} \mathrm{1,3-PDO}$ with a productivity of $2.6 \mathrm{~g} /$ (L.h) [8]. The microbial consortium DL38 with $95.57 \%$ abundance of Klebsiella pneumoniae achieved a relatively high 1,3-PDO titer of $81.4 \mathrm{~g} / \mathrm{L}$ with a yield of $0.52 \mathrm{~g} / \mathrm{g}$ [9]. The effect of initial $\mathrm{pH}$ on a batch mixed culture fermentation of glycerol was also investigated [10]. And the results indicated that the highest 1,3-PDO production yield of $0.64 \mathrm{~mol} / \mathrm{mol}$ was obtained at $\mathrm{pH} 7$ and 8 by the predominant bacteria from Clostridiaceae, Enterococcaceae, and Enterobacteriaceae families.

Furthermore, an anaerobic microbial consortium C2-2M enriched from anaerobic activated sludge with 94.64\% abundance of Clostridium butyricum produced 60.61 and $82.66 \mathrm{~g} / \mathrm{L}$ 1,3-PDO in batch and fedbatch fermentation, with the productivity of 3.79 and $3.06 \mathrm{~g} /($ L.h), respectively [7]. Performances of continuous fermentations were also investigated by consortium C2-2M and the highest 1,3-PDO production of $57.86 \mathrm{~g} / \mathrm{L}$ was achieved with a productivity of $5.55 \mathrm{~g} /(\mathrm{L} \cdot \mathrm{h})$ at a dilution rate of $0.096 \mathrm{~h}^{-1}$ and an initial glycerol concentration of $130 \mathrm{~g} / \mathrm{L}$ [11]. Furthermore, under micro-aerobic conditions, a novel consortium DUT08 primarily comprised of a strictly anaerobic microbe of $C$. butyricum, facultative microbes of $K$. pneumoniae and $E$. coli could efficiently convert crude glycerol to 1,3-PDO [4]. In a batch fermentation without nitrogen supply, $43.20 \mathrm{~g} / \mathrm{L} \mathrm{1,3-PDO}$ with a yield of $0.39 \mathrm{~g} / \mathrm{g}$ and a productivity of $0.98 \mathrm{~g} /($ L.h) was obtained.

Despite the fact that these natural microbial consortia could efficiently convert crude glycerol to 1,3-PDO, there are still significant limitations in answering the fundamental ecological and evolutionary questions, as well as the interaction mechanism surrounding natural microbial consortia. Even the simplest natural microbial consortia characterized to date contain tens to thousands of species, making it difficult to experimentally verify which species in such characterizations are actively part of the community or perform key functions [13]. The creation of artificial microbial consortia that retain the key features of their natural counterparts is a promising way to overcome the difficulties associated with studying natural communities. The construction of synthetic microbial consortia enables generation of defined systems with reduced complexity.

In our previous work, 1,3-PDO producing microbes of anaerobic $C$. butyricum DUT1 and facultative $K$. pneumoniae DUT2 were isolated from natural microbial consortium DUT08 [4]. In this study, the synthetic microbial consortia was successfully constructed and developed for 1,3-PDO production from crude glycerol. Co-culture systems containing a strict anaerobic microbe of $C$. butyricum and facultative microbes of $K$. pneumoniae and/or E. coli were investigated in order to improve the high oxygen tolerance of the strict anaerobes under micro-aerobic conditions. Furthermore, metabolic flux distribution and abundance of synthetic microbial consortia were discussed.

\section{Results And Discussion}

Comparison of 1,3-PDO production between mono- and co-culture of C. butyricum or/and K. pneumoniae 
C. butyricum (CB) and K. pneumoniae (KP), having a significant ability of 1,3-PDO production, attracted much attention. In this study, we chose a CB:KP co-culture to take advantage of these two 1,3-PDO producing microorganisms. K. pneumoniae is a facultative strain that can grow in aerobic, micro-aerobic, and anaerobic conditions on glycerol. As a result, we hope that the growth of $K$. pneumoniae will create an anaerobic environment that will benefit the growth of $C$. butyricum. The production of 1,3-PDO using crude glycerol under the mono-culture and the CB:KP co-culture at anaerobic serum vial level was tested, and the results are presented in Table 1. C. butyricum can grow well with $0.2 \mathrm{vvm}$ nitrogen supply for 3 min, yielding $10.79 \mathrm{~g} / \mathrm{L} 1,3-P D O$ with a yield to glycerol of $0.54 \mathrm{~g} / \mathrm{g}$. The mono-culture of $C$. butyricum produced no 1,3-PDO in the absence of $\mathrm{N}_{2}$. At different inoculation ratios, The CB:KP co-culture produced nearly identical amounts of 1,3-PDO. After $24 \mathrm{~h}$ cultivation without $\mathrm{N}_{2}, 6.84 \mathrm{~g} / \mathrm{L}$ 1,3-PDO was produced at the optimal ratio of 3:2. The level of 1,3-PDO production in the CB:KP co-culture was comparable to that in the KP mono-culture but lower than that in the $\mathrm{CB}$ mono-culture. Many byproducts were produced for the KP mono-culture and the CB:KP co-culture, including acetic acid, lactic acid, succinic acid, and ethanol. Butyric acid, on the other hand, was not detected in the CB:KP co-culture. It showed that dissolved oxygen in the medium inhibited the growth of $C$. butyricum in the CB:KP co-culture, even though K. pneumoniae was inoculated to create the anaerobic condition. K. pneumoniae was primarily responsible for the ability of the $\mathrm{CB}: \mathrm{KP}$ co-culture to produce 1,3-PDO without $\mathrm{N}_{2}$ supply. The performance of $K$. pneumoniae in producing 1,3-PDO under micro-aerobic conditions was inferior to that of $C$. butyricum under anaerobic conditions. As a result, the synthetic microbial consortium of $C$. butyricum and $K$. pneumoniae under micro-aerobic condition showed a weak 1,3-PDO production ability that was not comparable to the $\mathrm{CB}$ mono-culture under anaerobic condition.

Table 1

Comparison of 1,3-PDO production by the mono-culture and the CB:KP co-culture at different inoculation ratios under anaerobic serum vial level $(20 \mathrm{~g} / \mathrm{L}$ initial glycerol $)$

\begin{tabular}{|llllllll|}
\hline Microorganisms & RG & \multicolumn{7}{c|}{ Products $(\mathbf{g} / \mathbf{L})$} \\
\cline { 7 - 10 } & $(\mathbf{g} / \mathbf{L})$ & $\mathbf{1 , 3 - P D O}$ & Butyrate & Acetate & Lactate & Succinate & Ethanol \\
\hline C. butyricum DUT1* & 0.35 & 10.79 & 2.49 & - & 0.23 & - & - \\
\hline C. butyricum DUT1 & 19.88 & - & - & - & - & - & - \\
\hline K. pneumoniae DUT3 & 1.25 & 6.72 & - & 1.03 & 2.16 & 0.71 & 1.09 \\
\hline Syn-CB:KP (4:1) & 0.78 & 6.41 & - & 0.56 & 1.72 & 0.32 & 0.89 \\
\hline Syn-CB:KP (3:2) & 1.56 & 6.84 & - & 0.43 & 2.31 & 0.34 & 0.91 \\
\hline Syn-CB:KP (2:3) & 1.13 & 6.62 & - & 0.48 & 2.41 & 0.36 & 1.00 \\
\hline Syn-CB:KP (1:4) & 1.27 & 6.67 & - & 0.56 & 2.46 & 0.35 & 0.82 \\
\hline *Anaerobic condition: 0.2 vvm N 2 supply for 3 min; RG: Residual glycerol; -: not detectable. \\
\hline
\end{tabular}


Effect of E. coli addition on mono-culture of CB and co-culture of CB and KP for 1,3-PDO production under non- $\mathrm{N}_{2}$ aeration conditions

E. coli $(\mathrm{EC})$, a microbe that does not produce 1,3-PDO but consumes oxygen, was inoculated to the monoculture and co-culture cultivation to improve 1,3-PDO production under micro-aerobic conditions. To this end, the results obtained for 1,3-PDO by different synthetic microbial consortia are represented in Fig. 1.

The results showed that $E$. coli could significantly increase 1,3-PDO production in the CB:EC co-culture at different inoculation ratios when compared to the $\mathrm{CB}$ mono-culture without $\mathrm{N}_{2}$ supply. Glycerol was almost depleted after $24 \mathrm{~h}$ of fermentation in the $\mathrm{CB}$ mono-culture and the CB:EC co-culture with $0.2 \mathrm{vvm}$ $\mathrm{N}_{2}$ supply. In the CB:EC co-culture with a 1:1 inoculation ratio and no $\mathrm{N}_{2}$ supply, $16.38 \mathrm{~g} / \mathrm{L}$ glycerol remained in the fermentation media, and only $1.72 \mathrm{~g} / \mathrm{L}$ 1,3-PDO was produced within $24 \mathrm{~h}$. Glycerol was nearly depleted in the CB:EC co-culture after $48 \mathrm{~h}$ of fermentation in the absence of $\mathrm{N}_{2}$. The results showed that the presence of $E$. coli causes $C$. butyricum to grow and produce 1,3-PDO slowly in the absence of $\mathrm{N}_{2}$. The fermentation time for the CB:EC co-culture was extended under micro-aerobic conditions (no $\mathrm{N}_{2}$ supply), and the yield of 1,3-PDO to glycerol decreased by $24 \%$ at the inoculation ratio of 1:1 when compared to anaerobic conditions ( $0.2 \mathrm{vvm} \mathrm{N}_{2}$ supply). Once the anaerobic condition was established by $E$. coli, the $\mathrm{CB}: \mathrm{EC}$ co-culture could efficiently produce 1,3-PDO regardless of the CB:EC inoculation ratios. At a $\mathrm{CB}: \mathrm{EC}$ inoculation ratio of 3:2, the optimal 1,3-PDO concentration of $8.48 \mathrm{~g} / \mathrm{L}$ with a yield to glycerol of $0.42 \mathrm{~g} / \mathrm{g}$ was obtained. Furthermore, for both the $\mathrm{CB}$ mono-culture and the CB:EC coculture, butyric acid was the main byproduct, followed by acetic acid.

The metabolic profile of the KP:EC co-culture was similar to that of the KP mono-culture. Byproducts included lactic acid, succinic acid, acetic acid, and ethanol. Among these byproducts, lactic acid had the highest concentration. When EC was inoculated to the CB:KP co-culture, butyric acid was produced as the main byproduct instead of lactic acid, indicating that anaerobic cultivation conditions were formed and $C$. butyricum could grow well in the $\mathrm{CB}: \mathrm{KP}: \mathrm{EC}$ co-culture. In comparison to the $\mathrm{CB}: \mathrm{KP}$ co-culture, the $\mathrm{CB}: \mathrm{KP}: \mathrm{EC}$ co-culture demonstrated a greater ability to remove oxygen due to $E$. coli inoculation. As a result, the highest 1,3-PDO concentration of $7.02 \mathrm{~g} / \mathrm{L}$ was obtained in the CB:KP:EC co-culture at a CB:KP:EC inoculation ratio of 13:1:1. The CB:KP:EC co-culture reduced the final 1,3-PDO concentration compared to the $\mathrm{CB}$ :EC co-culture because $C$. butyricum and $K$. pneumoniae competed for glycerol to produce 1,3-PDO. The ability of KP to produce 1,3-PDO is weaker than the ability of $\mathrm{CB}$, and $\mathrm{KP}$ is more competitive than $\mathrm{CB}$ under micro-aerobic conditions, making KP to become the dominant in the $\mathrm{CB}: \mathrm{KP}: \mathrm{EC}$ co-culture.

\section{Metabolic profile and dynamic abundance of synthetic microbial consortia in batch fermentation under non-strictly anaerobic conditions}

In our previous study, natural microbial consortium DUT08 isolated from wastewater samples could efficiently covert crude glycerol to 1,3-propanediol (1,3-PDO) under anaerobic and micro-aerobic conditions. Because of the coexistence of facultative $K$. pneumoniae and strictly anaerobic $C$. butyricum, 
the organism compositions in DUT08 changed during the long-term preservation process. Furthermore, it was difficult to investigate and determine the function of other unidentified organisms in consortium DUT08, which had a low abundance of $2.20 \%$. As a result, construction and maintaining a stable, controlled and robust microbial consortium is critical for efficient 1,3-PDO production. In this case, we constructed and investigated various synthetic microbial consortia composed of strictly anaerobic $C$. butyricum, facultative $K$. pneumoniae, and facultative $E$. coli with or without $\mathrm{N}_{2}$ supply to efficiently produce 1,3-PDO. the co-production of 1,3-PDO, butyric acid and lactic acid by the synthetic co-culture under batch fermentation at micro-aerobic and anaerobic conditions was investigated, and the results are shown in Fig. 2.

C. butyricum grew well, and no substrate inhibition was observed. At an initial glycerol concentration of $106.01 \mathrm{~g} / \mathrm{L}$ and $0.20 \mathrm{vvm} \mathrm{N}_{2}$ supply, an excellent glycerol consumption rate of $3.55 \mathrm{~g} /(\mathrm{L} . \mathrm{h})$ was obtained (Fig. 2A). As a result, $47.43 \mathrm{~g} / \mathrm{L} \mathrm{1,3-PDO} \mathrm{with} 7.45 \mathrm{~g} / \mathrm{L}$ butyric acid, $5.73 \mathrm{~g} / \mathrm{L}$ acetic acid, and $2.48 \mathrm{~g} / \mathrm{L}$ lactic acid were produced by the $\mathrm{CB}$ mono-culture under $0.2 \mathrm{vvm} \mathrm{N}_{2}$ supply. The yield and productivity of 1,3-PDO was $0.45 \mathrm{~g} / \mathrm{g}$ and $1.61 \mathrm{~g} /$ (L.h), respectively. Furthermore, C. butyricum did not grow under 0.02 vvm $\mathrm{N}_{2}$ supply for $3 \mathrm{~h}$ at the same initial glycerol concentration, implying that a strictly anaerobic environment did not developed. It has been reported that $C$. butyricum exhibits substrate inhibition at initial glycerol concentration greater than $70 \mathrm{~g} / \mathrm{L}$ [14]. The same inhibition was also observed by Dietz et al. when mixed cultures were used [8]. In our previous study, obvious substrate inhibition was observed by C. butyricum at the initial glycerol of $125.18 \mathrm{~g} / \mathrm{L}$ [4]. C. butyricum grew well in this study, with no substrate inhibition occurred at an initial glycerol of $106.01 \mathrm{~g} / \mathrm{L}$ and a supply of $0.2 \mathrm{vvm} \mathrm{N}_{2}$. However, the cells ceased to grow when supplied with $0.02 \mathrm{vvm} \mathrm{N}_{2}$. The inhibition of dissolved oxygen was relieved once KP and EC were induced into the mono-culture system. Therefore, to obtain a high 1,3-PDO yield and productivity by $C$. butyricum, it is safe to keep the glycerol concentration below $110 \mathrm{~g} / \mathrm{L}$ and no dissolved oxygen in medium.

The performance of the KP mono-culture was also investigated and the results are presented in Fig. 2B. Although the final 1,3-PDO concentration obtained by the KP mono-culture with an initial glycerol of $110.09 \mathrm{~g} / \mathrm{L}$ was comparable to $C$. butyricum, K. pneumoniae demonstrated weaker glycerol consumption ability and lower 1,3-PDO productivity than $C$. butyricum. The fermentation lasted for $46 \mathrm{~h}$ with the KP mono-culture. The final 1,3-PDO concentration, yield, productivity, and glycerol consumption rate were $45.03 \mathrm{~g} / \mathrm{L}, 0.41 \mathrm{~g} / \mathrm{g}, 0.98 \mathrm{~g} /(\mathrm{L} . \mathrm{h})$, and $2.37 \mathrm{~g} /(\mathrm{L} . \mathrm{h})$, respectively. When compared to C. butyricum, 1,3-PDO yield, productivity, and glycerol consumption rate by $K$. pneumoniae decreased about $9 \%, 39 \%$, and $33 \%$, respectively. Lactic acid was obtained at a concentration of $25.92 \mathrm{~g} / \mathrm{L}$ as the main byproduct by $K$. pneumoniae. The critical concentration of lactic acid for inhibiting cell growth of $K$. pneumoniae was determined to be $19 \mathrm{~g} / \mathrm{L}$ under anaerobic conditions and $26 \mathrm{~g} / \mathrm{L}$ under aerobic conditions, respectively [15]. Obvious cell growth inhibition was also observed at $12.59 \mathrm{~g} / \mathrm{L}$ lactic acid after fermenting for $22 \mathrm{~h}$ by K. pneumoniae DUT2. Most $K$. pneumoniae produce 2,3-butanediol as a byproduct, and the similarity of 2,3-butanediol and 1,3-pandediol causes the separation barriers [16]. K. pneumoniae DUT2 shows unique characters from other $K$. pneumoniae strains in that no 2,3-butanediol is produced during the 
fermentation process. In a $5 \mathrm{~L}$ bioreactor, the dynamic dissolved oxygen and oxidation-reduction potential (ORP) variation of the KP mono-culture during the fermentation process was studied. In the KP monoculture, dissolved oxygen was quickly depleted within $0.5 \mathrm{~h}$. ORP decreased from $-250 \mathrm{mv}$ to $-580 \mathrm{mv}$ in 8 $\mathrm{h}$, then remained at $-580 \mathrm{mv}$ for $15 \mathrm{~h}$ before increasing to $-500 \mathrm{mv}$ at the end of fermentation. According to the findings, $K$. pneumoniae scavenged oxygen and could create an anaerobic environment for $C$. butyricum growth in order to produce 1,3-PDO.

When KP and CB are co-cultured, KP and CB compete for glycerol consumption to produce 1, 3-PDO, while $\mathrm{KP}$ provides an anaerobic environment for $\mathrm{CB}$ growth. However, in this study, KP's ability to produce 1,3PDO from glycerol is weaker than CB's. As a result, if the KP abundance in mix culture is too high, the final 1, 3-PDO concentration and yield to glycerol will be low. In order for $\mathrm{CB}$ to grow and produce 1,3-PDO efficiently in the CB: KP co-culture, KP abundance should be kept to a low level. In our pervious study, natural microbial consortium DUT08 showed efficient conversion of crude glycerol to 1,3-PDO at an initial abundance of $85.25 \%$ Clostridium, $12.54 \%$ Escherichia, and $0.007 \%$ Klebsiella [4]. Therefore, the initial abundance of $\mathrm{CB}$ in the synthetic microbial consortia was guaranteed to be at least $85 \%$. In the CB:KP coculture with initial inoculation ratio of $92.5: 7.5,52.08 \mathrm{~g} / \mathrm{L}$ 1,3-PDO with a yield of $0.49 \mathrm{~g} / \mathrm{g}$, a productivity of $1.80 \mathrm{~g} /(\mathrm{L} . \mathrm{h})$ was obtained at an initial glycerol of $108.79 \mathrm{~g} / \mathrm{L}$ with $0.02 \mathrm{vvm} \mathrm{N}_{2}$ supply. Furthermore, byproducts such as $7.14 \mathrm{~g} / \mathrm{L}$ butyric acid, $2.93 \mathrm{~g} / \mathrm{L}$ lactic acid, and $6.39 \mathrm{~g} / \mathrm{L}$ acetic acid were generated (Fig. 2C). No CB growth was observed under the same cultivation conditions as the CB mono-culture. $\mathrm{CB}$ accounted for $92.50 \%$ of the initial abundance, while KP accounted for $7.50 \%$ (Fig. 3). Within $5 \mathrm{~h}$, the KP ratio increased to $28.81 \%$, while the $\mathrm{CB}$ ratio decreased to $71.19 \%$. Following that, the abundance of $\mathrm{CB}$ increased to $87.17 \%$ within $10 \mathrm{~h}$ and remained at a high level of $93.41 \%$ at $14 \mathrm{~h}$.

The EC mono-dynamic culture's dissolved oxygen and oxidation-reduction potential (ORP) variation was also investigated. Under $0.02 \mathrm{vvm} \mathrm{N}_{2}$ supply, dissolved oxygen was quickly exhausted in the EC monoculture within $10 \mathrm{~min}$. ORP decreased from $-280 \mathrm{mv}$ to $-590 \mathrm{mv}$ in $4 \mathrm{~h}$, then remained at $-590 \mathrm{mv}$ for $5 \mathrm{~h}$, increasing to $-400 \mathrm{mv}$ at $12.5 \mathrm{~h}(20 \mathrm{~g} / \mathrm{L}$ glucose used). The results indicated that $E$. coli scavenged oxygen more quickly than $K$. pneumoniae and provided a anaerobic environment for $C$. butyricum growth to produce 1,3-PDO faster. In the CB:EC co-culture with initial inoculation ratio of 92.5:7.5, $51.33 \mathrm{~g} / \mathrm{L}$ 1,3PDO with a yield of $0.49 \mathrm{~g} / \mathrm{g}$, a productivity of $1.47 \mathrm{~g} /(\mathrm{L} . \mathrm{h})$ was obtained at an initial glycerol of 107.18 $\mathrm{g} / \mathrm{L}$ with $0.02 \mathrm{vvm} \mathrm{N}_{2}$ supply (Fig. 2D). Compared to the CB:EC co-culture, KP inoculation in the CB monoculture can enhance glycerol utilization rate and improve 1,3-PDO productivity. The abundance of synthetic $\mathrm{CB}: \mathrm{EC}$ co-culture was also investigated and the results indicated that the abundance of $\mathrm{EC}$ declined from $7.50-3.23 \%$ within $5 \mathrm{~h}$, and only $0.83 \%$ at $15 \mathrm{~h}$. The abundance of CB increased from 92.5-99.17\% within $15 \mathrm{~h}$ (Fig. 3).

In the synthetic CB:KP:EC co-culture with initial inoculation ratio of 85:7.5:7.5, $51.43 \mathrm{~g} / \mathrm{L} \mathrm{1,3-PDO}$ with a yield of $0.49 \mathrm{~g} / \mathrm{g}$, a productivity of $1.77 \mathrm{~g} /(\mathrm{L} . \mathrm{h})$ was obtained at an initial glycerol of $107.18 \mathrm{~g} / \mathrm{L}$ with 0.02 vvm $\mathrm{N}_{2}$ supply (Fig. 2E). Furthermore, the main byproducts were $7.77 \mathrm{~g} / \mathrm{L}$ butyric acid, $6.03 \mathrm{~g} / \mathrm{L}$ acetic acid, and $3.29 \mathrm{~g} / \mathrm{L}$ lactic acid, resulting in a total product yield to glycerol of $0.66 \mathrm{~g} / \mathrm{g}$. As shown in Fig. 3 , 
the abundance of synthetic $\mathrm{CB}: \mathrm{KP}: \mathrm{EC}$ indicated that $\mathrm{CB}$ was always the dominant bacterium, accounting for over $80 \%$ of the total abundance for the whole fermentation.

The metabolic flux distribution of the products under different cultures was examined as shown in Fig. 4. For the KP mono-culture, the metabolic flow was mainly distributed to 1,3-PDO and lactic acid pathway which occupied about $65.06 \%$ of the whole glycerol metabolism. For the CB mono-culture under strictly anaerobic condition, $52.14 \%$ of glycerol metabolic flow was distributed to 1,3-PDO and butyric acid pathway. The results of the synthetic CB:KP, CB:EC, and CB:KP:EC co-cultures showed that strictly anaerobic and facultative bacteria can coexist under micro-aerobic conditions. The highest 1,3-PDO flux of $49.17 \%$ was obtained by the synthetic CB:KP:EC co-culture while $7.43 \%, 5.77 \%, 3.15 \% 4.24 \%$ and $2.13 \%$ of flux was distributed to butyric acid, acetic acid, lactic acid, ethanol and succinic acid pathway. Synthetic microbial consortia can all efficiently convert crude glycerol to produce 1,3-PDO and achieve high 1,3-PDO yield. The ability to produce 1,3-PDO is dependent on the abundance of 1,3-PDO producing bacteria that have a high 1,3-PDO production capacity as well as a high glycerol utilization capacity. In this study, a maximum 1,3-PDO concentration of $52.08 \mathrm{~g} / \mathrm{L}$ was obtained by the synthetic CB:KP coculture at an initial abundance ratio of $92.5: 7.5$, with a yield of $0.49 \mathrm{~g} / \mathrm{g}$ and a productivity of $1.80 \mathrm{~g} /(\mathrm{L} . \mathrm{h})$. Compared to the CB mono-culture under strictly anaerobic condition, the final 1,3-PDO concentration, yield, and productivity by the synthetic CB:KP consortia increased by $10 \%, 9 \%$, and $12 \%$, respectively. Compared to the KP mono-culture, the final 1,3-PDO concentration, yield, and productivity by the synthetic CB:KP consortia increased by $16 \%, 19 \%$, and $84 \%$, respectively. The results indicated that synthetic coculture established in this study improved 1,3-PDO production from crude glycerol significantly.

Several efforts were developed to improve 1,3-PDO production using co-cultivation. A waste lard utilizing Pseudomonas alcaligenes PA-3 and a 1,3-PDO producing Klebsiella pneumoniae AA405 were cocultivated by using waste lard as the sole carbon source [17]. In bioreactor, the co-cultivation system produced $5.98 \mathrm{~g} / \mathrm{L} \mathrm{1,3-PDO} \mathrm{and} \mathrm{4.29} \mathrm{g/L} \mathrm{2,3-butanediol} \mathrm{(2,3-BD)} \mathrm{from} 100 \mathrm{~g} / \mathrm{L}$ waste lard within $72 \mathrm{~h}$, and the conversion rate of 1,3-PDO and 2,3-BD from waste lard were $62.95 \%$ and $0.75 \%$, respectively. Moreover, the control of redox balances in co-culture fermentation of Geobacter sulfurreducens and Clostridium pasteurianum was studied (Moscoviz et al., 2017). As a result, it was demonstrated that $G$. sulfurreducens could grow with $C$. pasteurianum as sole electron acceptor. The metabolic pattern of $C$. pasteurianum was significantly altered in favor of improved 1,3-PDO and butyric acid production ( $+37 \%$ and $+38 \%$ resp.) at the expense of butanol and ethanol production ( $-16 \%$ and $-20 \%$ resp.). A novel mixed culture comprising $C$. butyricum and a methane bacterium, Methanosarcina mazei, to relieve the inhibition and to utilize the by-products for energy production was investigated by metabolic modelling [18]. The calculations revealed that the presence of methanol can increase methane production by $130 \%$. M. mazei can consume more than $70 \%$ of the acetate secreted by $C$. butyricum.

\section{Performance and dynamic abundance of synthetic microbial consortia in fed-batch fermentation under non- strictly anaerobic conditions}


As previously stated, a high initial glycerol concentration inhibits cell growth and reduces glycerol conversion yield. As a result, fed-batch cultivation by various synthetic consortia was developed and compared to evaluate consortia performance. As shown in Fig. 5, obvious cell growth inhibition was observed in the $\mathrm{CB}$ mono-culture with $0.02 \mathrm{vvm} \mathrm{N}_{2}$ supply, which lasted for at least $12 \mathrm{~h}$. The synthetic $\mathrm{CB}: \mathrm{KP}: \mathrm{EC}$ co-culture exhibited high cell density due to the inoculation of KP and EC. When compared to the synthetic consortia, the natural consortium DUT08 showed similar cell growth to the KP mono-culture and a weak 1,3-PDO production capability. There was only $44.29 \mathrm{~g} / \mathrm{L}$ 1,3-PDO produced, with a yield of $0.36 \mathrm{~g} / \mathrm{g}$ and a productivity of $0.94 \mathrm{~g} /(\mathrm{L} . \mathrm{h})$. The results were very similar to those of the KP mono-culture indicating that $K$. pneumoniae replaced $C$. butyricum as the dominant bacterium in natural consortium DUT08. The final butyric acid concentration of $1.75 \mathrm{~g} / \mathrm{L}$ and lactic acid concentration of $22.91 \mathrm{~g} / \mathrm{L}$ obtained by DUT08 also verified this deduction. The results presented here are consistent with those presented in the previous section, indicating that the $C B$ inoculation ratio was an important parameter in the development of synthetic consortia capable of efficiently producing 1,3-PDO from crude glycerol. In our previous report, $C$. butyricum was the dominant bacterium accounting for $85.25 \%$ of the proportion in DUT08 [4]. As a result, a higher 1,3-PDO concentration of $58.33 \mathrm{~g} / \mathrm{L}$ was produced, with a yield of 0.52 $\mathrm{g} / \mathrm{g}$ and total productivity of $1.94 \mathrm{~g} /(\mathrm{L} . \mathrm{h})$ at an initial glycerol concentration of $117.01 \mathrm{~g} / \mathrm{L}$. Due to the coexistence of facultative bacteria and strictly anaerobic bacteria, the preservation conditions for DUT08 are very strict in order to maintain the bacteria ratio. The varied composition of consortium DUT08 makes the 1,3-PDO production unstable. Therefore, maintaining a stable and controlled composition of organisms within a co-culture is essential and important for efficient 1,3-PDO production.

The stronger 1,3-PDO formation capability was obtained by synthetic consortia of CB:KP, CB:EC, and $\mathrm{CB}: K P: E C$ co-cultures. Among the three constructed synthetic consortia, synthetic CB:KP:EC showed the maximum 1,3-PDO concentration of $77.68 \mathrm{~g} / \mathrm{L}$ which is $30 \%$ higher than the production by the CB monoculture at $0.02 \mathrm{vvm} \mathrm{N}_{2}$ supply. A higher 1,3-PDO concentration of $81.39 \mathrm{~g} / \mathrm{L}$ was obtained by the synthetic CB:KP:EC consortium under $0.2 \mathrm{vvm} \mathrm{N}_{2}$ supply. However, the 1,3-PDO yield decreased from $0.51 \mathrm{~g} / \mathrm{g}$ to $0.49 \mathrm{~g} / \mathrm{g}$ when $\mathrm{N}_{2}$ supply increased from $0.02 \mathrm{vvm}$ to $0.2 \mathrm{vvm}$. When synthetic consortia were used, butyric acid was the main byproduct. Synthetic CB:KP:EC co-culture yielded over $17 \mathrm{~g} / \mathrm{L}$ butyric acid. Moreover, the synthetic CB:EC co-culture and CB:KP co-culture produced $14.57 \mathrm{~g} / \mathrm{L}$ and $15.60 \mathrm{~g} / \mathrm{L}$ butyric acid, respectively. Because of the high concentration of butyric acid produced, the synthetic consortia are primarily composed of $C$. butyricum, which produces butyric acid. Lactic acid was produced as the primary byproduct by using the natural consortium DUT08 and the KP mono-culture. Lactic acid concentrations in the natural consortium DUT08 and the KP mono-culture are $22.91 \mathrm{~g} / \mathrm{L}$ and $20.81 \mathrm{~g} / \mathrm{L}$, respectively.

As shown in Fig. 6, the abundance of CB in synthetic CB:EC, CB:KP, and CB:KP:EC co-culture accounted for $92.5 \%, 92.5 \%$, and $85.0 \%$ at the beginning, respectively. The abundance of KP increased to $23.61 \%$ and $20.10 \%$ within $4 \mathrm{~h}$ in synthetic CB:KP and CB:KP:EC co-culture under $0.02 \mathrm{vvm} \mathrm{N}_{2}$ supply, respectively. The results indicated that CB growth was inhibited for the first $4 \mathrm{~h}$ in above two synthetic consortia. Subsequently, the abundance of CB started to increase to $91.82 \%$ and $89.71 \%$ within $8 \mathrm{~h}$, and remained 
high at $89.27 \%$ and $90.27 \%$ at $12 \mathrm{~h}$, respectively. Within $10 \mathrm{~min}, \mathrm{E}$. coli created an anaerobic condition for the $\mathrm{CB}$ :EC co-culture under $0.02 \mathrm{vvm} \mathrm{N}_{2}$ supply. As a result, the abundance of $\mathrm{CB}$ has always been greater than $85 \%$.

\section{Conclusions}

In this study, the synthetic microbial consortia were constructed by adding facultative microbe of $K$. pneumoniae DUT2 and/or E. coli DUT3 into strict anaerobic microbe of $C$. butyricum DUT1 culture under micro-aerobic conditions. The interaction of commensalism and competition resulted in synthetic microbial consortia that could efficiently convert crude glycerol to 1,3-PDO even under micro-aerobic conditions. In a batch fermentation, the maximum 1,3-PDO titer of $52.08 \mathrm{~g} / \mathrm{L}$, with a yield of $0.49 \mathrm{~g} / \mathrm{g}$ and a productivity of $1.80 \mathrm{~g} /($ L.h) were obtained by synthetic CB:KP co-culture at an initial abundance ratio of 92.5:7.5. Compared to the CB mono-culture under strictly anaerobic condition, the final 1,3-PDO concentration, yield, and productivity by the synthetic CB:KP consortia increased $10 \%, 9 \%$, and $12 \%$, respectively. The highest 1,3-PDO flux of $49.17 \%$ was obtained by the synthetic CB:KP:EC co-culture at an initial abundance ratio of $85: 7.5: 7.5$ while $7.43 \%, 5.77 \%, 3.15 \% 4.24 \%$ and $2.13 \%$ of flux was distributed to butyric acid, acetic acid, lactic acid, ethanol and succinic acid pathway. In a fed-batch fermentation, synthetic CB:KP:EC co-culture demonstrated maximum 1,3-PDO concentration of $77.68 \mathrm{~g} / \mathrm{L}$ with a yield of $0.51 \mathrm{~g} / \mathrm{g}$ which is $30 \%$ and $13 \%$ higher than the production by the $\mathrm{CB}$ mono-culture at $0.02 \mathrm{vvm} \mathrm{N}_{2}$ supply. The results presented here indicating that the $C B$ inoculation ratio was an important parameter in the development of synthetic consortia capable of efficiently producing 1,3-PDO from crude glycerol.

\section{Materials And Methods}

\section{Strains and medium}

C. butyricum DTU1, K. pneumoniae DTU2 and E. coli DTU3 were all isolated from natural microbial consortium DUT08, which was enriched and isolated from wastewater in sewage treatment of petrochemical company [4]. C. butyricum DTU1 was anaerobically pre-cultured in a serum bottle containing $100 \mathrm{~mL}$ nitrogen-gassed sterilized seed medium at $200 \mathrm{rpm}$ and $37^{\circ} \mathrm{C}$ for $12 \mathrm{~h} . \mathrm{K}$. pneumoniae DTU2 and E. coli DTU3 were aerobically pre-cultured in a serum bottle containing $100 \mathrm{~mL}$ sterilized seed medium at $200 \mathrm{rpm}$ and $37^{\circ} \mathrm{C}$ for $12 \mathrm{~h}$, respectively. Seed and fermentation media, as well as crude glycerol used were described as our previous report [4]. Crude glycerol concentration given in this study indicated the absolute glycerol content of the solution regardless of the impurities.

\section{Culture conditions}

The synthetic microbial consortia were inoculated to the fermentation medium to determined their ability to convert crude glycerol to 1,3-PDO. The volume ratio was used in all of the inoculation ratios. Before inoculation, the seed cultures were diluted to a similar OD value to maintain a constant inoculation volume. Shake flask culture was carried out in $250 \mathrm{~mL}$ serum bottles with media volume of $200 \mathrm{~mL}$. The 
batch and fed-batch fermentations were carried out in a $5 \mathrm{~L}$ bioreactor with the working volume of $2 \mathrm{~L}$ at $37^{\circ} \mathrm{C}$ and $200 \mathrm{rpm}$. The inoculum volume for synthetic consortia was $10 \%(\mathrm{v} / \mathrm{v})$. The $\mathrm{pH}$ was controlled at 7.0 automatically adding $5 \mathrm{~mol} / \mathrm{L} \mathrm{NaOH}$. Continuous feeding was started in fed-batch fermentations when the residue glycerol concentration was less than $20 \mathrm{~g} / \mathrm{L}$, and the feeding rate was adjusted to keep the glycerol concentration between 20 and $30 \mathrm{~g} / \mathrm{L}$. Anaerobic fermentation was performed with $0.2 \mathrm{vvm}$ $\mathrm{N}_{2}$ supply for $1 \mathrm{~h}$ before and $2 \mathrm{~h}$ after inoculation. Micro-aerobic fermentation was achieved with or without $0.02 \mathrm{vvm} \mathrm{N}_{2}$ supply for $1 \mathrm{~h}$ before and $2 \mathrm{~h}$ after inoculation. Samples were taken at regular intervals to analyze biomass, glycerol and products concentration. The metabolic flux distributions of the products were calculated by dividing the product formation rates (qp) by the glycerol uptake rate (qs) and multiplying by 100 .

\section{qPCR analysis}

The bacterial compositions in synthetic microbial consortia were analyzed by quantitative Real-time PCR. Genomic DNA was extracted according to the manufacturer's instructions (TaKaRa MiniBest Bacterial Genome DNA Extraction Kit Ver. 3.0). The purity of DNA was checked by using a NanoDrop ND-2000 spectrophotometer (NanoDrop technologies, Wilmington, DE) and electrophoresis. Primers were designed according to $16 \mathrm{~S}$ rRNA sequence difference among $C$. butyricum DUT1, K. pneumoniae DUT2 and E. coli DUT3 listed in Table 2. The reaction mixture for each assay contained $10 \mu \mathrm{L}$ of SYBR Premic EX Taq (TaKaRa Bio technology Co., Dalian, China), $0.8 \mu \mathrm{L}$ of each primer, $0.4 \mu \mathrm{L}$ of ROX Reference Dye II, and 2 $\mu \mathrm{L}(20 \mathrm{ng})$ of genomic DNA. The PCR was performed using the following protocol: $95^{\circ} \mathrm{C}$ for $30 \mathrm{~s}$, following by 40 cycles at $95^{\circ} \mathrm{C}$ for $3 \mathrm{~s}$, and $60^{\circ} \mathrm{C}$ for $30 \mathrm{~s}$. The assay was performed at least three times for each sample. To generate standard curves for quantification, three standard plasmids were used. Standard plasmid was constructed by pESI-T vector ligation with PCR product of 16 S rRNA, and then transformed to E. coli DH5a. Copy number was calculated as follows.

Copy number $=\frac{\text { DNA amount }}{\text { DNA length } \times 660 \times 1 \times 10^{9}} \times 6.022 \times 10^{23}$

Table 2

Primers designed for $C$. butyricum DUT1, K. pneumoniae DUT2 and $E$. coliDUT3

\begin{tabular}{|lll|}
\hline Primer & Sequences $\left(\mathbf{5}^{\prime} \mathbf{3}^{\prime} \mathbf{3}^{\prime}\right)$ & Targeted strain \\
\hline HgF & AAGAAGCTTTAGAAGATCCTAA & C. butyricum DUT1 \\
\hline HgR & GGACAACATGAGGTAAACATTG & C. butyricum DUT1 \\
\hline phoEF & TGCCCAGACCGATAACTTTA & K. pneumoniae DUT2 \\
\hline phoER & CTGTTTCTTCGCTTCACGG & K. pneumoniae DUT2 \\
\hline$y b b W F$ & TGATTGGCAAAATCTGGCCG & E. coliDUT3 \\
\hline$y b b W R$ & GAAATCGCCCAAATCGCCAT & E. coliDUT3 \\
\hline
\end{tabular}

Page $11 / 19$ 
qPCR reactions were run on serial dilutions of each standard plasmid to relate threshold cycle number (Ct value) to copy numbers of the target sequence and to generate standard curves for quantification in unknown samples. Standard curves were linear across five orders of magnitude of $10^{2}-10^{7}$ copies with $R^{2}$ of 0.99-1.

\section{Analytical methods}

Biomass concentration was determined by the measurement of optical density at $650 \mathrm{~nm}\left(\mathrm{OD}_{650}\right)$. As previously described, glycerol and its products (1,3-PDO, butyric acid, acetic acid, lactic acid, succinic acid, and ethanol) were analyzed using HPLC (Waters 600E) [4]. Sample solutions were diluted to suitable concentrations and filtered through a $0.22 \mu \mathrm{m}$ membrane filter before injection.

\section{Abbreviations}

1,3-PD0: 1,3-propanediol; CB: Clostridium butyricum DUT1; KP: Klebsiella pneumoniae DUT2; EC: Escherichia coli DUT3; PTT: Polytrimethylene terephthalate; RG: Residual glycerol;

\section{Declarations}

\section{Ethical approval and consent to participate}

Not applicable.

\section{Consent for publication}

All authors approved the consent for publishing the manuscript to Biotechnology for Biofuels.

\section{Availability of data and materials}

The datasets used and/or analyzed during the current study are available from the corresponding author on reasonable request.

\section{Competing interests}

The authors declare that they have no competing interests.

\section{Funding}

This work was supported by the National Natural Science Foundation of China [grant number 22078043].

\section{Authors' contributions}

YQS advised on the experiments design, analyzed results and drafted this manuscript. LYL, YFZ and JDH designed and carried out the experiments. XZL revised the manuscript. All authors read and approved the final manuscript. 


\section{References}

1. Brenner K, You LC, Arnold FH. Engineering microbial consortia: a new frontier in synthetic biology. Trends Biotechnol. 2008;26:483-9.

2. Navarrete-Bolanos JL. Improving traditional fermented beverages: How to evolve from spontaneous to directed fermentation. Eng Life Sci. 2012;12:410-8.

3. Sun YQ, Xu ZZ, Zheng YF, Zhou JJ, Xiu ZL. Efficient production of lactic acid from sugarcane molasses by a newly microbial consortium CEE-DL15. Process Biochem. 2019;81:132-8.

4. Sun YQ, Zheng YF, Wang XL, Zhou JJ, Xiu ZL. Fermentation performance and mechanism of a novel microbial consortium DUT08 for 1,3-propandiol production from biodiesel-derived crude glycerol under non-strictly anaerobic conditions. Process Biochem. 2019;83:27-34.

5. Kleerebezem R, van Loosdrecht MC. Mixed culture biotechnology for bioenergy production. Curr Opin Biotechnol. 2007;18:207-12.

6. Sabra W, Dietz D, Tjahjasari D, Zeng AP. Biosystems analysis and engineering of microbial consortia for industrial biotechnology. Eng Life Sci. 2010;10:407-21.

7. Zhou JJ, Shen JT, Jiang LL, Sun YQ, Mu Y, Xiu ZL. Selection and characterization of an anaerobic microbial consortium with high adaptation to crude glycerol for 1,3-propanediol production. Appl Microbiol Biotechnol. 2017; 101:5985-96.

8. Dietz D, Zeng AP. Efficient production of 1,3-propanediol from fermentation of crude glycerol with mixed cultures in a simple medium. Bioprocess Biosyst Eng. 2014;37:225-33.

9. Jiang LL, Liu HF, Mu Y, Sun YQ, Xiu ZL. High tolerance to glycerol and high production of 1,3propanediol in batch fermentations by microbial consortium from marine sludge. Eng Life Sci. 2017;17:635-44.

10. Moscoviz R, Trably E, Bernet N. Consistent 1,3-propanediol production from glycerol in mixed culture fermentation over a wide range of pH. Biotechnol Biofuels. 2016;9:32.

11. Zhou JJ, Shen JT, Wang XL, Sun YQ, Xiu ZL. Stability and oscillatory behavior of microbial consortium in continuous conversion of crude glycerol to 1,3-propanediol. Appl Microbiol Biotechnol. 2018;102:8291-305.

12. Wang XL, Zhou JJ, Sun YQ, Xiu ZL. Bioconversion of raw glycerol from waste cooking-oil-based biodiesel production to 1,3-propanediol and lactate by a microbial consortium. Front Bioeng Biotechnol. 2019;7:14.

13. Grosskopf T, Soyer OS. Synthetic microbial communities. Curr Opin Microbiol. 2014;18:72-7.

14. Szymanowska-Powalowska D. The effect of high concentrations of glycerol on the growth, metabolism and adaptation capacity of Clostridium butyricum DSP1. Electron J Biotechnol. 2015;18: 128-33.

15. Cheng KK, Liu HJ, Liu DH. Multiple growth inhibition of Klebsiella pneumoniae in 1,3-propanediol fermentation. Biotechnol Lett. 2005;27:19-22. 
16. Sun YQ, Shen JT, Yan L, Zhou JJ, Jiang LL, Chen Y, Yuan JL, Feng EM, Xiu ZL. Advances in bioconversion of glycerol to 1,3-propanediol: Prospects and challenges. Process Biochem. 2018;71:134-46.

17. Li Y, Zhu S, Ge X. Co-production of 1,3-propanediol and 2,3-butanediol from waste lard by cocultivation of Pseudomonas alcaligenes and Klebsiella pneumoniae. Curr microbiol. 2019;76: 41524.

18. Bizukojc M, Dietz D, Sun JB, Zeng AP. Metabolic modelling of syntrophic-like growth of a 1,3propanediol producer, Clostridium butyricum, and a methanogenic archeon, Methanosarcina mazei, under anaerobic conditions. Bioprocess Biosyst Eng. 2010;33:507-23.

\section{Figures}

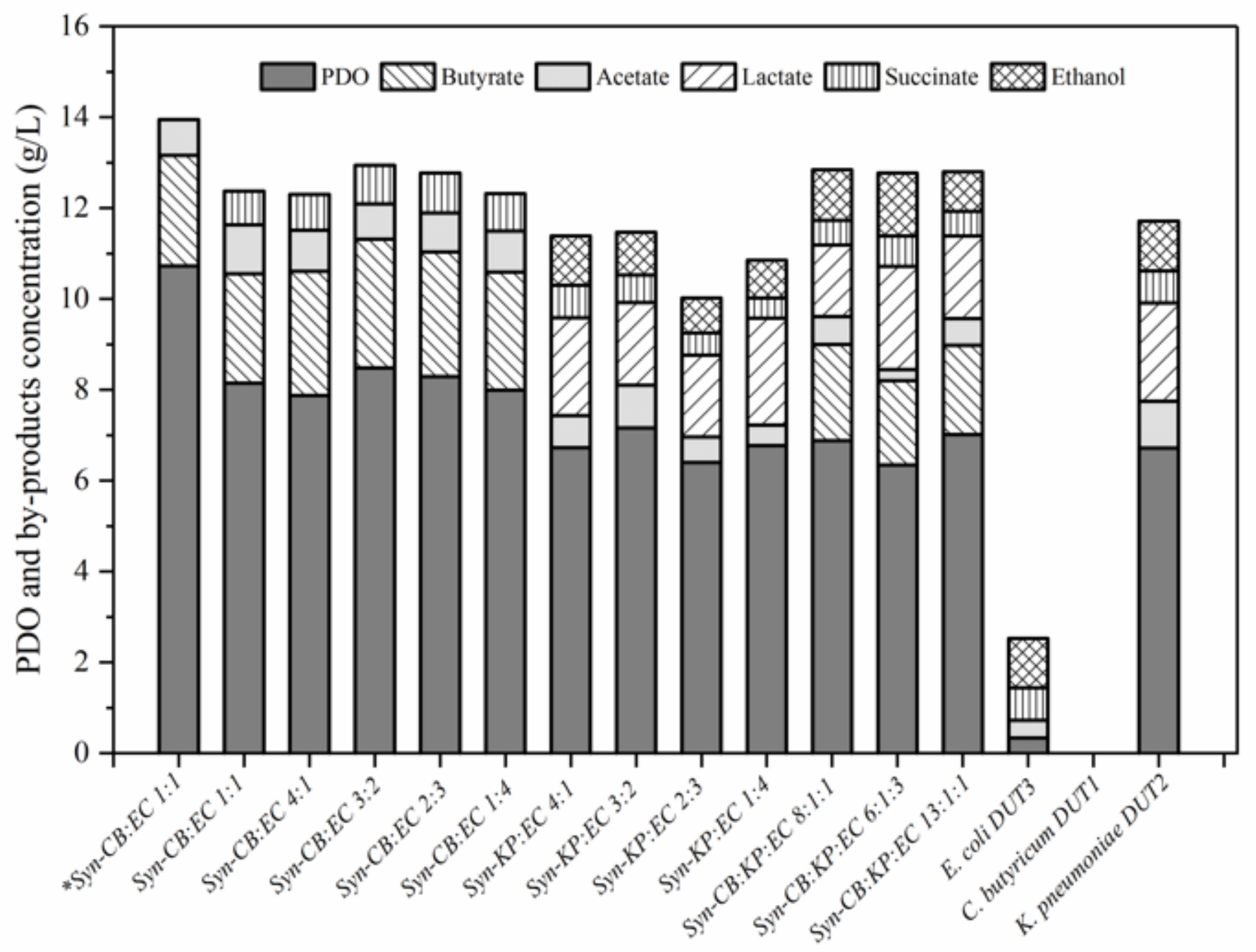

Figure 1

Comparison of 1,3-PDO and byproducts production by the mono-culture and the co-culture at different inoculation ratios without N2 supply. (*Anaerobic condition: $0.2 \mathrm{vvm} \mathrm{N2}$ supply for $3 \mathrm{~min}$ ) 


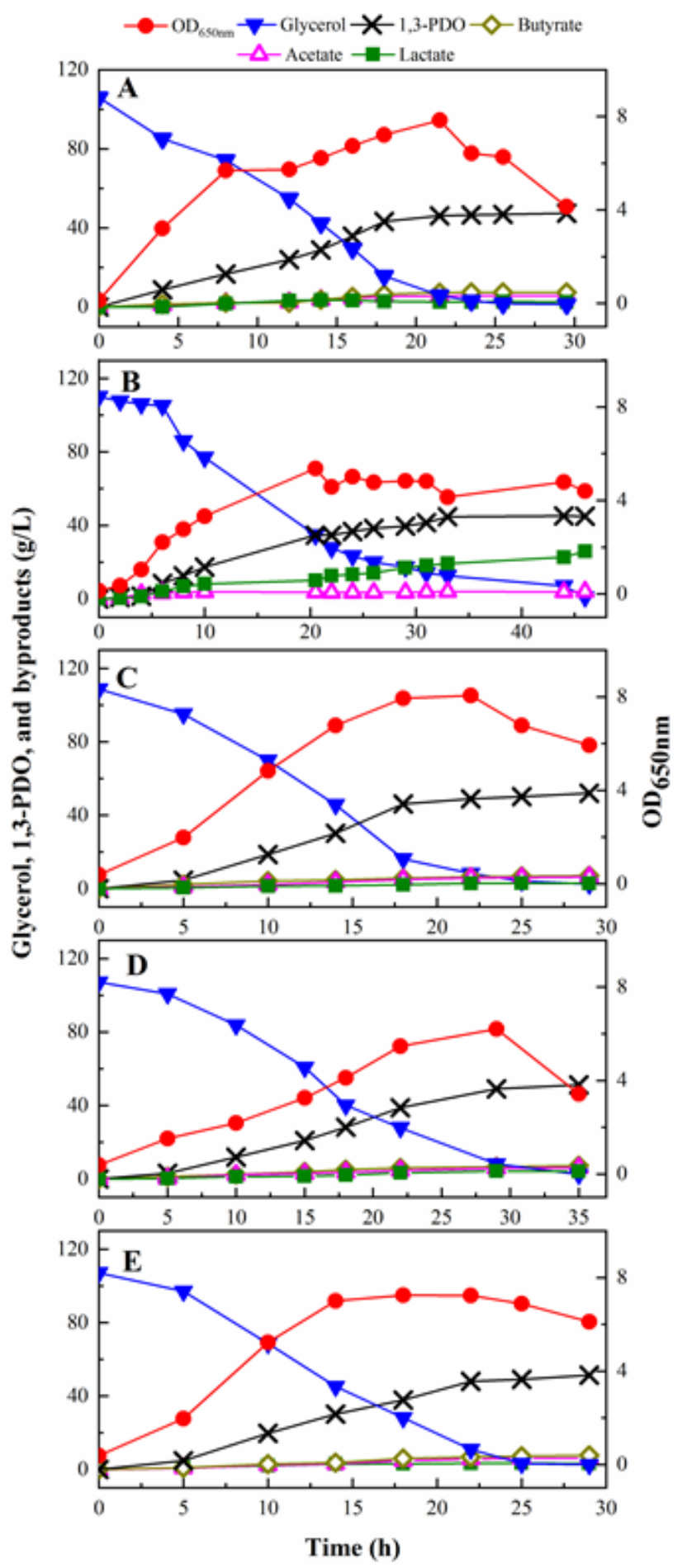

\section{Figure 2}

Fermentation performance of mono-culture and synthetic microbial consortia in batch fermentation. (A) The CB mono-culture with N2 supply at 0.2 vvm for $3 \mathrm{~h}$; (B) The KP mono-culture without N2 supply; (C) The CB:KP co-culture with N2 supply at $0.02 \mathrm{vvm}$ for $3 \mathrm{~h}$; (D) The CB:EC co-culture with N2 supply at 0.02 vvm for $3 \mathrm{~h}$; (E) The CB:KP:EC co-culture with N2 supply at 0.02 vvm for $3 \mathrm{~h}$. Fermentation was carried out at $37 \mathrm{oC}, \mathrm{pH} 7.0$ and $200 \mathrm{rpm}$ in $5 \mathrm{~L}$ bioreactor containing $2 \mathrm{~L}$ medium. 


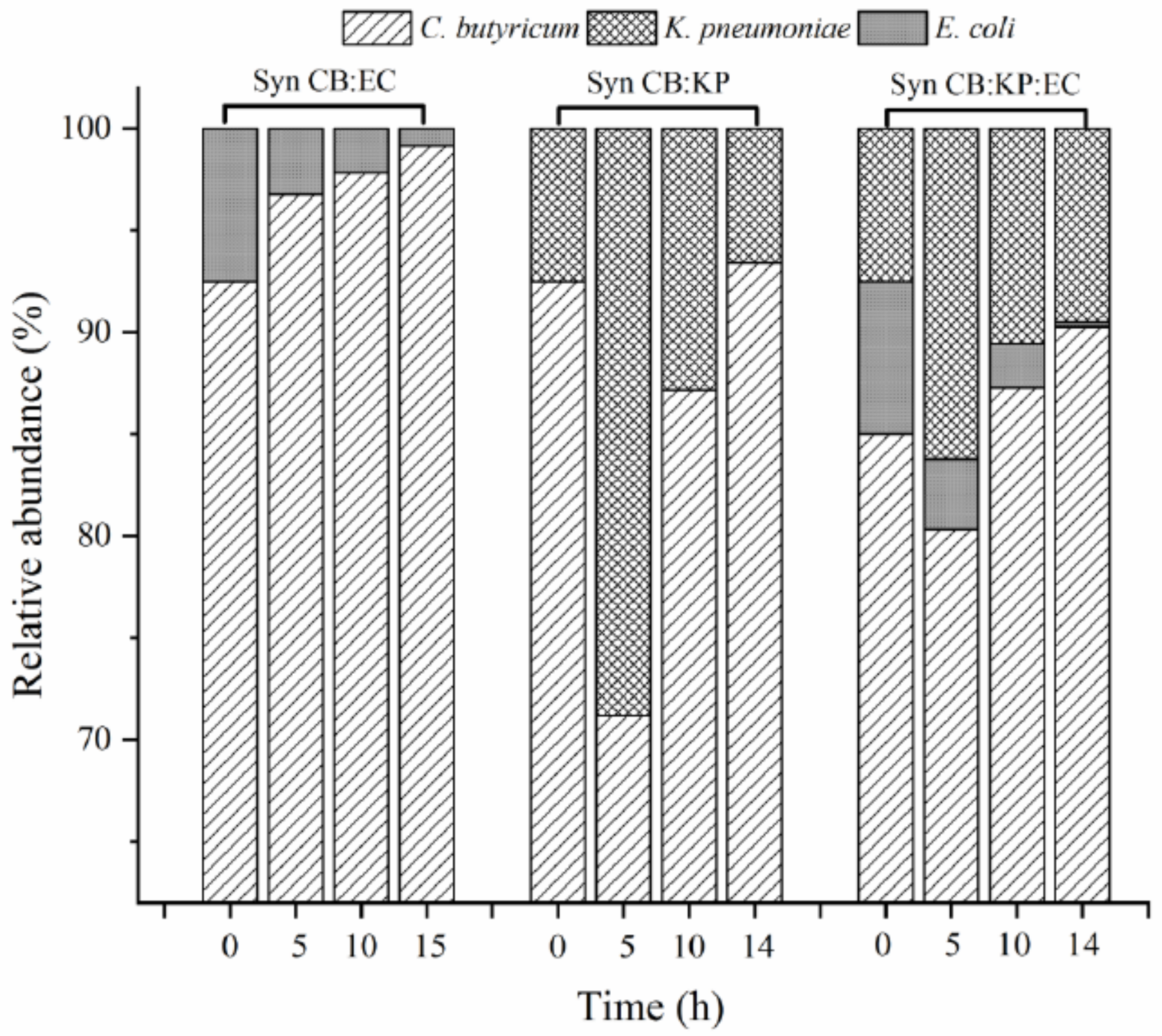

Figure 3

Abundance of synthetic microbial consortia during the batch fermentation at $0.02 \mathrm{vvm} \mathrm{N2}$ supply for $3 \mathrm{~h}$ 
$45.30^{\mathrm{a}} 1,3-\mathrm{PDO} \longleftarrow$ Glycerol $\longrightarrow$ Biomass

$0^{\mathrm{b}}$

$41.29^{c}$

$49.00^{\mathrm{d}}$

$49.03^{\mathrm{e}}$

$49.17^{f}$
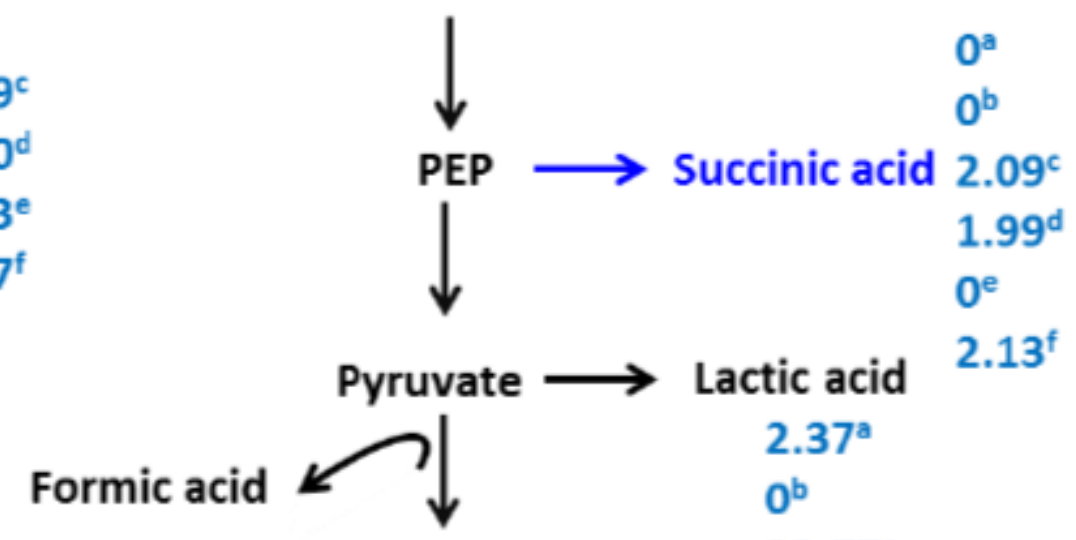

\begin{tabular}{|c|c|c|}
\hline \multicolumn{2}{|c|}{ Acetyl-CoA } & \multirow{4}{*}{$\begin{array}{r}23.77 \\
2.76^{\mathrm{d}} \\
3.96^{\mathrm{e}} \\
3.15^{\mathrm{f}} \\
\text { Acetic acid }\end{array}$} \\
\hline & & \\
\hline & & \\
\hline Butyric acid & Ethanol & \\
\hline $7.11^{\mathrm{a}}$ & $0^{\mathrm{a}}$ & $5.47^{\mathrm{a}}$ \\
\hline $0^{\mathrm{b}}$ & $0^{\mathrm{b}}$ & $0^{b}$ \\
\hline $0^{c}$ & $3.35^{\mathrm{c}}$ & $3.65^{\mathrm{c}}$ \\
\hline $6.72^{d}$ & $3.12^{\mathrm{d}}$ & $6.01^{\mathrm{d}}$ \\
\hline $6.99^{\mathrm{e}}$ & $4.32^{e}$ & $5.82^{\mathrm{e}}$ \\
\hline $7.43^{f}$ & $4.24^{f}$ & $5.77^{f}$ \\
\hline
\end{tabular}

$\rightarrow$ Represents the common metabolic pathway of CB and KP

$\rightarrow$ Represents the metabolic pathway of KP

Represents the metabolic pathway of CB

Figure 4

The flux distribution of glycerol metabolism at different mono-culture and synthetic co-culture in batch fermentation. aThe CB mono-culture with $\mathrm{N} 2$ supply at 0.2 vvm for $3 \mathrm{~h}$; bThe $\mathrm{CB}$ mono-culture with N2 supply at 0.02 vvm for $3 \mathrm{~h}$; CThe KP mono-culture without N2 supply; dThe CB:KP co-culture with N2 supply at $0.02 \mathrm{vvm}$ for $3 \mathrm{~h}$; eThe CB:EC co-culture with $\mathrm{N} 2$ supply at $0.02 \mathrm{vvm}$ for $3 \mathrm{~h}$; fThe CB:KP:EC coculture with $\mathrm{N} 2$ supply at $0.02 \mathrm{vvm}$ for $3 \mathrm{~h}$. 

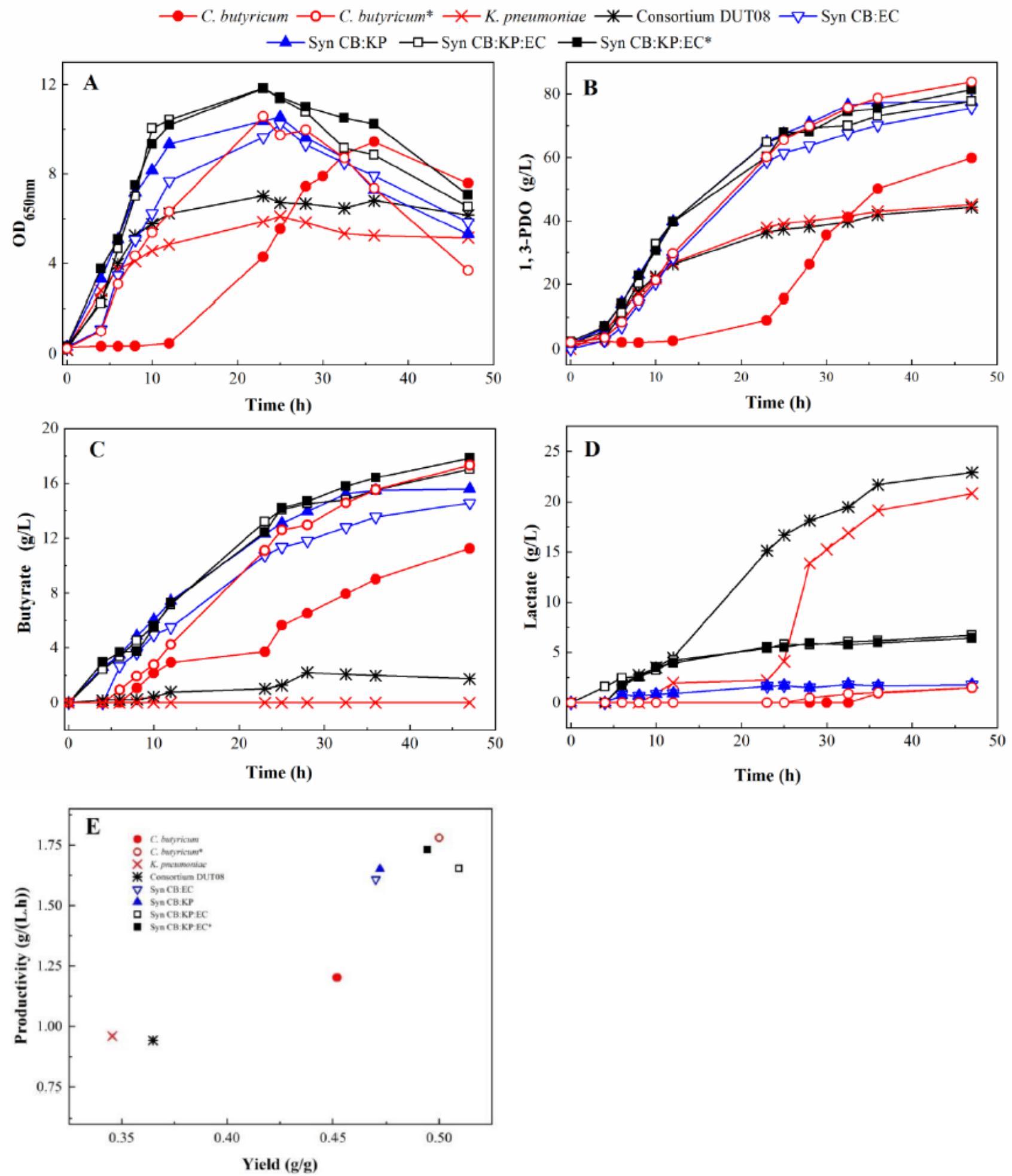

Figure 5

Fermentation performance of the mono-culture and the synthetic microbial consortia at $0.02 \mathrm{vvm} \mathrm{N2}$ supply for $3 \mathrm{~h}$ under fed-batch fermentation (A)OD650nm; (B) 1,3-PDO concentration; (C) Butyrate concentration; (D) Lactate concentration; (E) Yield and productivity. Fermentation was carried out at 37 $\mathrm{oC}$, pH 7.0 and $200 \mathrm{rpm}$ in $5 \mathrm{~L}$ bioreactor containing $2 \mathrm{~L}$ medium. Residual glycerol controlled around 40 $\mathrm{g} / \mathrm{L}$. * represented $0.2 \mathrm{vvm} \mathrm{N2}$ supply for $3 \mathrm{~h}$. 


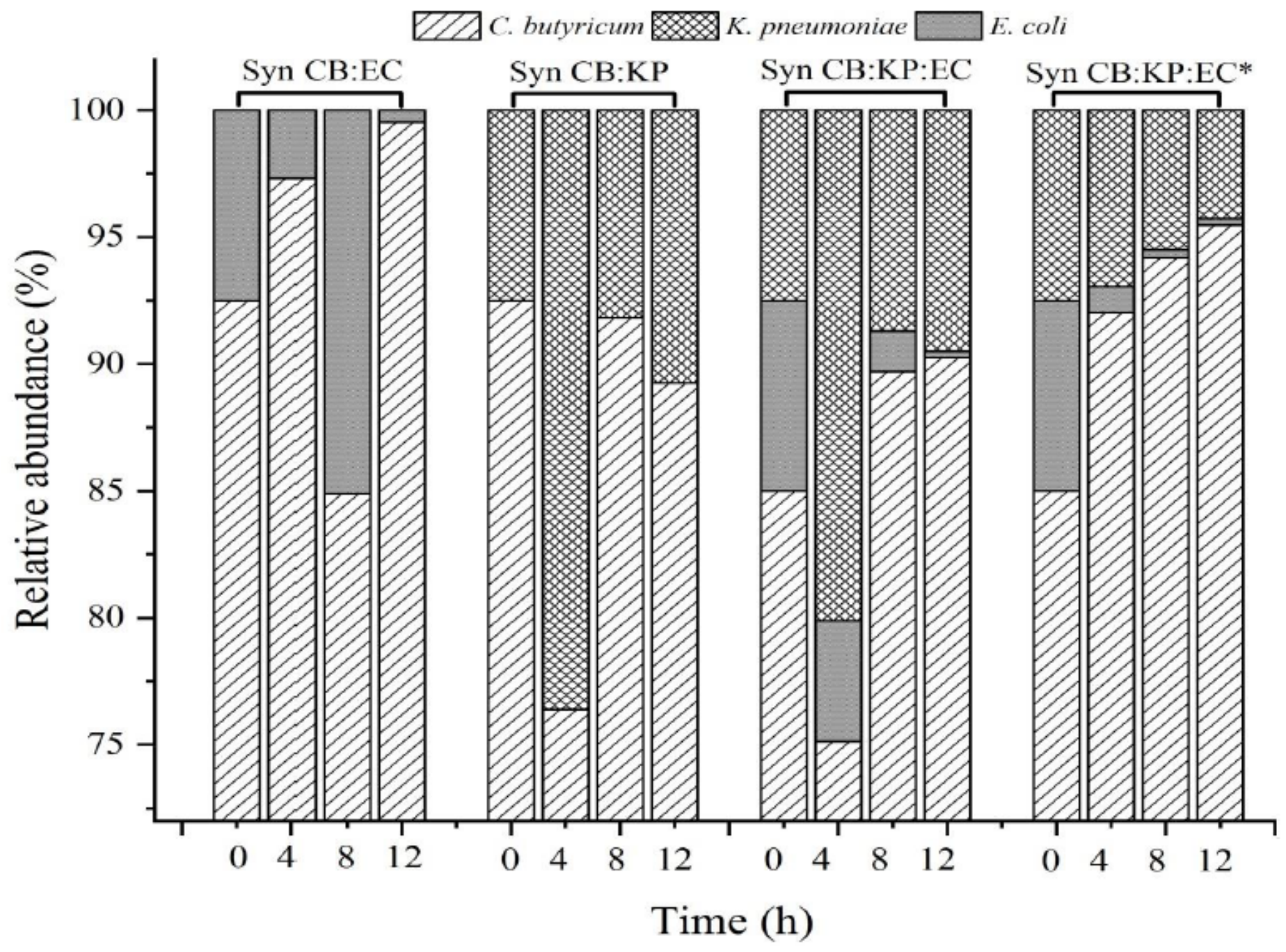

Figure 6

Abundance of synthetic microbial consortia during the fed-batch fermentation process at $0.02 \mathrm{vvm} \mathrm{N2}$ supply for $3 \mathrm{~h}$ 